\title{
Research Status of Defrosting Technology of Air Source Heat Pump
}

\author{
Cheng Kun ${ }^{1}$, Liu Bingzhan ${ }^{1}$ and Li Guangpeng ${ }^{1 *}$ \\ ${ }^{1}$ Shandong Institute of Commerce \& Technology, Jinan, Shandong 250103, China
}

\begin{abstract}
Air source heat pump has the characteristics of high efficiency, energy saving, green and pollution-free, and has been widely used in building energy saving projects. However, the frosting phenomenon of the air source heat pump evaporator during the heating operation in cold areas in winter will reduce the heat production of the system, affect the comfort experience, and even cause the system down or irreversible damage to the heat pump system when the frosting is serious. This paper summarizes the research of domestic and foreign scholars on air source heat pump defrosting, analyzes the existing problems of defrosting technology, and finally looks forward to the future development trend of air source heat pump defrosting research.
\end{abstract}

\section{Introduction}

Air source heat pump has the characteristics of high efficiency, energy saving, dual use of cooling and heating, etc, and it is widely used in the fields of heating and air conditioning[1]. When the air source heat pump is running in low temperature area, once the surface temperature of the outdoor heat exchanger is lower than zero, and lower than the dew point temperature of the outdoor air, the surface of the outdoor heat exchanger will frost. With the increase of frost thickness, the additional heating resistance on the surface of outdoor heat exchanger increases gradually, which weakens the flow heat transfer between refrigerant and outdoor air[2]. At the same time, frosting makes the air flow resistance between the heat exchange fins increase, thus greatly reducing the heat pump heating power and heating coefficient. It will also increase the energy consumption of the fan, leading to further deterioration of the operating condition of the air source heat pump, and serious accidents will occur. Therefore, it is of great significance to take appropriate methods for periodic defrosting of outdoor heat exchangers to ensure that the air source heat pump still has good operating efficiency in low temperature areas and to popularize the air source heat pump technology.

\section{Overseas and domestic research status}

For the frosting problem of air source heat pump operating in low temperature areas, domestic and foreign scholars have done a lot of research on defrosting. The current main methods include reverse cycle defrosting, hot refrigerant by-pass defrosting, heating defrosting, energy storage defrosting, external force defrosting and active defrosting.

\subsection{Reverse cycle defrosting}

Reverse circulation defrosting is the use of the four-way reversing valve to switch the heat pump from the heating mode to the refrigeration mode[3], at this time the indoor heat exchanger and outdoor heat exchanger interchange, through the refrigerant heat release to melt the frost layer on the outdoor heat exchanger, defrosting after the end of the recovery mode. When using reverse circulation defrost, the system only needs to switch the four-way directional valve, without adding additional devices and auxiliary heat source. When using reverse circulation defrost, the system only needs to switch the four-way directional valve, without adding additional devices and auxiliary heat source. This method has the advantages of simple operation, low cost and good applicability. It is widely used in large, medium and small heat pump defrosting, and is also one of the most widely used defrosting methods at present.

\subsection{Hot refrigerant by-pass defrosting}

The defrosting method is arranged between the compressor outlet and the entrance of the outdoor heat exchanger by the bypass pipeline[4]. The system directly introduces the high temperature and high pressure exhaust from the compressor into the outdoor heat exchanger and makes the frost layer melt by the heat of the high temperature refrigerant itself. When the hot air bypass defrost is removed, the pressure of the system changes smoothly because the four-way directional valve does not need to change direction. Therefore, there will be no airflow noise, and the impact on the unit is small.

*Corresponding author's e-mail: LB14020028@cumt.edu.cn 
At the same time, the hot refrigerant bypass method in the whole energy of defrosting process required from the compressor housing accumulating heat compressor work, don't need to indoor heat can also give a small amount of indoor heat, indoor temperature fluctuation is small, good comfort, also applies to large, medium and small heat pump defrosting, is currently one of the widely used methods of defrosting.

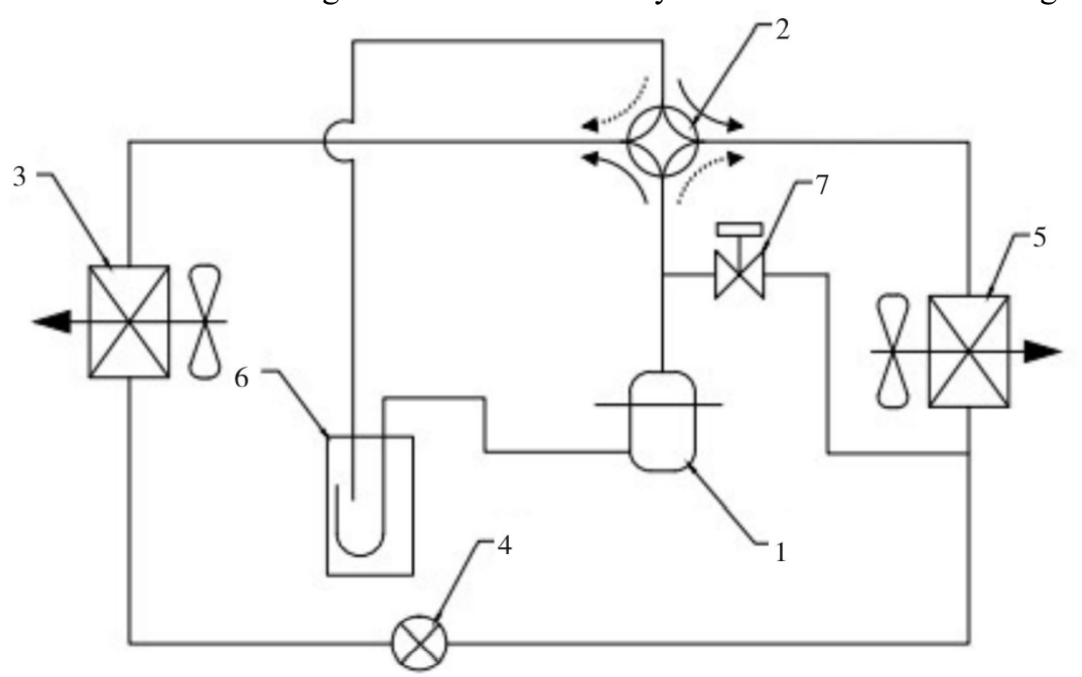

1-Compressor. 2-Four-way valve. 3-Indoor heat exchanger. 4-Throttle valve. 5-Outdoor heat exchanger. 6-Gas-liquid separator. 7 Hot refrigerant bypass solenoid valve

Figure 1. Schematic diagram of reverse cycle defrosting and hot refrigerant by-pass defrosting

\subsection{Heating defrosting}

Heated defrosting mainly includes electric heating defrosting and solar energy assisted defrosting two.[5] Using electric energy or solar energy as defrosting energy, the outdoor heat exchanger inlet air or refrigerant working medium is heated, in order to improve the evaporation temperature and compressor inlet refrigerant temperature, to achieve the purpose of defrosting or delay the frosting.

The electric heating defrost has the advantages of simple system, thorough defrost and easy control, so it is more suitable for small heat pump system. The solar energy is used to assist defrosting. The defrosting energy is clean and renewable, and it is easy to realize rational allocation of thermal energy level. It is suitable for areas with abundant solar energy resources.

\subsection{Energy storage defrosting}

Energy storage defrost method is put forward on the basis of reverse cycle defrost.[6] The heat storage device is combined with the air source heat pump, part of the heat is stored in the heat storage device during the system heating operation, and then the heat is released during defrosting.

Energy storage defrosting method fundamentally solves the problem of insufficient energy source of traditional thermal defrosting method, and improves the reliability and stability of the operation of air source heat pump to a certain extent. It is a kind of defrosting method which is widely studied at present.

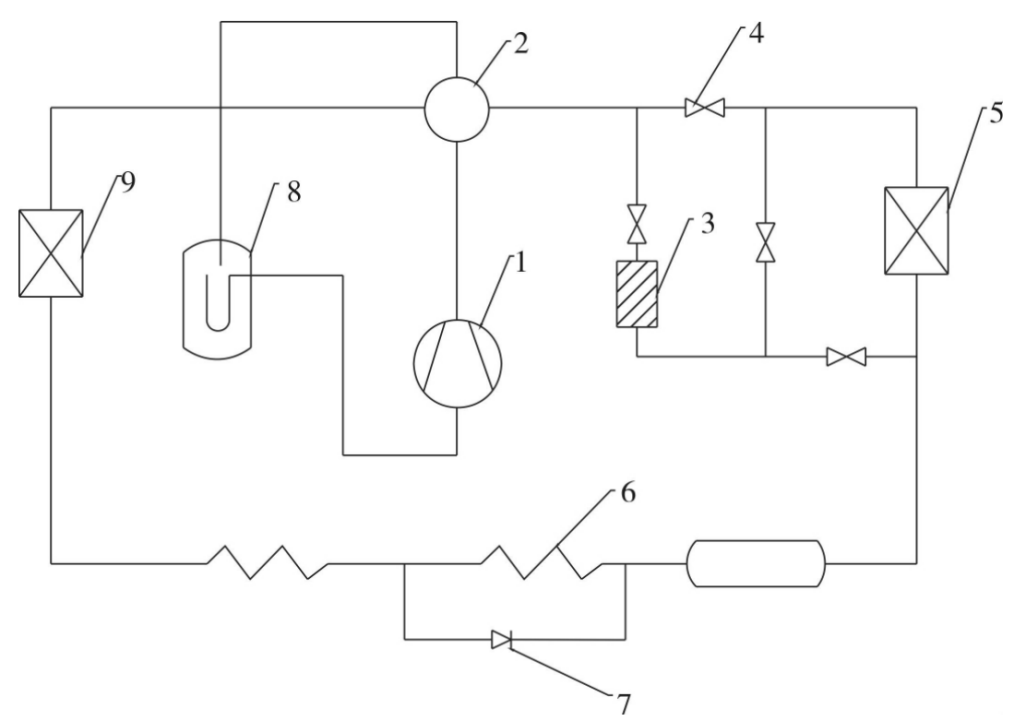

1-Compressor. 2-Four-way valve. 3-Heat storage heat exchanger. 4-Solenoid valve. 5-Indoor heat exchanger. 6-Capillary throttle. 7Check valve. 8-Gas-liquid separator. 9-Outdoor heat exchangers

Figure 2. Diagram of an air source heat pump heat storage and defrosting system 


\subsection{External force defrosting}

Defrost can be carried out by increasing external force field, including external electric field, magnetic field and ultrasonic wave[7]. The external electric and magnetic fields mainly influence the frost crystal growth through electric or magnetic forces. Frost crystal in the growth process by the external force tensile become slender, fragile, resulting in the surface of the outdoor heat exchanger adhesion reduced, easy to fall off. Ultrasonic defrosting is mainly through the application of appropriate frequency of ultrasonic and frost resonance effect, the use of resonance to destroy the frost layer, to achieve the purpose of defrosting.

\subsection{Active defrosting}

In addition to the above defrosting methods, through the study of the frosting mechanism, the researchers found that the method of actively suppressing the frosting of the outdoor heat exchanger can fundamentally solve the frosting problem of the air source heat pump. The current research is mainly to control the surface frosting of outdoor heat exchanger by changing the system flow.

\section{Existing problems}

In conclusion, the research of defrosting technology promotes the application of air source heat pump in low temperature area and promotes the development of air source heat pump. Throughout the research of scholars at home and abroad, the use of heating defrosting, reverse cycle defrosting or hot air by-pass defrosting, energy storage defrosting, increasing external force field and other defrosting technologies are effective ways to solve the problem of air source heat pump operating under low temperature conditions. However, there are still some problems in these defrosting technologies through the analysis of the research status of air source heat pump defrosting at home and abroad.

The primary problem of reverse cycle defrosting is the lack of defrosting energy and long defrosting time. Defrost energy only compressor work and indoor heat exchanger surface waste heat, with the indoor heat exchanger surface temperature decreases, the evaporation temperature will also drop, resulting in low pressure cut off or wet compression problems, causing serious damage to the compressor. When the four-way valve is reversing, the noise is large, which will cause a certain impact on the system and affect the performance and service life of the unit. Defrosting process will interrupt heating, resulting in large fluctuations in indoor temperature, indoor thermal comfort decline.

The hot refrigerant by-pass defrosting has the problems of low inspiratory superheat and long defrosting time. Due to the single source of defrosting energy and the large energy loss in the process of defrosting, the defrosting time is longer than that of reverse cycle defrosting. In the process of defrosting, the low suction superheat leads to the low exhaust and suction temperature of the compressor, and too long defrosting time will cause harm to the safe operation of the compressor.

The biggest disadvantage of electric heating defrosting is that the energy consumption is too large, can only be used for small heat pumps, not for large and medium-sized heat pumps, which limits the use range of this technology. In the process of application, the high grade heat energy is degraded and utilized, resulting in unreasonable matching of heat energy levels.

Although energy storage and defrosting solve the problem of insufficient energy source for defrosting, there is still the problem of heat supply interruption during defrosting. In addition, due to the addition of heat storage device, the complexity of the system and the cost of the equipment increase. Moreover, at present, the energy storage defrost technology is still in the experimental research stage, and the actual products are very few, and the stability and reliability of its operation in the real application are still uncertain.

The defrosting effect of increasing external force field has been studied, but its defrosting mechanism is still in the stage of theoretical and experimental research. The applied electric field has certain danger and high energy consumption, but the ultrasonic defrosting can only remove the frosting in a certain area, and can not remove the base ice on the fin.

\section{Conclusions}

Frosting problem seriously restricts the vigorous development of air source heat pump. In order to improve the adaptability of air source heat pump in low temperature area, the researchers have done a lot of research on heat pump defrosting. Different defrosting technology has different advantages, and the formation of frost layer is directly related to the conditions of outdoor temperature and humidity, different regions, different locations and different periods of temperature and humidity changes are different, which will affect the defrosting performance of the unit. Based on the summary of the research status at home and abroad, this paper analyzes the problems existing in the current defrosting technology, and prospects the development trend of air source heat pump defrosting research in the future.

At present, air source heat pump defrosting technology still has some shortcomings, how to further improve the traditional defrosting technology and improve the defrosting efficiency and reliability still need to be further studied to solve. The technology of energy storage and defrosting has made some achievements, but it can not be widely used in practice. How to obtain the heat storage installation with large heat storage capacity, small occupation area and good economy still needs to be studied in depth. The applied electric field, magnetic field or ultrasonic is a new defrosting technology, which has a good development prospect. However, the defrosting mechanism and how to apply external force on the outdoor heat exchanger remain to be further studied. 
The frost suppression technology provides some ideas for new defrosting methods. From the source of unit design, it is advisable to optimize the structure design of outdoor evaporator. From the aspect of unit transformation, coating should be used to suppress frost. These two defrosting means have high practical value, scholars are still carrying out experimental and theoretical research, looking forward to large-scale industrialization.

Multi-mode defrost method is another research idea after the bottleneck of defrost research technology, which is mainly realized by local organic combination of existing technology. From the perspective of life cycle, the heat source, control and post-processing in the whole process of defrosting were optimized to maximize the defrosting performance. With the development of current science and technology, multi-mode defrosting technology is constantly developing and progressing, which urgently needs to be further promoted.

\section{Acknowledgments}

Thanks to all the teachers of the Heat Pump Technical team of our school, we can guarantee the normal production of the prototype of defrosting technology of air source heat pump.

\section{References}

1. Ji Y J, Bae H H, Lee S J, et al. (2013) Continuous heating of an air-source heat pump during defrosting and improvement of energy efficiency, Applied Energy, 110( 5):9-16.

2. Song Mengjie, Liu Shengchun, Deng Shiming, etal.(2017) Experimental investigation on reverse cycle defrosting performance improvement for an ASHP unit by evenly adjusting the refrigerant distribution in its outdoor coil, Applied Thermal Engineering, 114:611-620.

3. Jiankai Dong, Shiming Deng, Yiqiang Jiang, etal.(2012) An experimental study on defrosting heat supplies and energy consumptions during a reverse cycle defrost operation for an air source heat pump, Applied Thermal Engineering, 37:380-387.

4. Kim J, Choi H J, Kim K C.(2015) A combined Dual Hot-Gas Bypass Defrosting method with accumulator heater for an air to air heat pump in cold region, Applied Energy, 147:344 -352.

5. Zhongbao Liu, Pengyan Fan, Qinghua Wang.(2018) Air source heat pump with water heater based on a bypass-cycle defrosting system using compressor casing thermal storage, Applied Thermal Engineering,128: 1420-1429.

6. Amer M, Wang C C. (2017) Review of defrosting methods. Renewable \& Sustainable Energy Reviews, 73:53-74.
7. S W Wang, Z Y Liu. (2005) A new method for preventing HP from frosting, Renewable Energy, 30(5):753- 761 . 